\title{
Efecto de Restricciones VaR sobre coberturas en mercados eléctricos
}

Recibido: 8 de septiembre de 2015 - Aceptado: 25 de septiembre de 2016

Doi: dx.doi.org/10.12804/revistas.urosario.edu.co/economia/a.5625

\author{
Alfredo Trespalacios Carrasquilla* \\ Juan Fernando Rendón García ${ }^{+}$ \\ Javier Orlando Pantoja Robayo ${ }^{\ddagger}$
}

Resumen

Se analiza el efecto que tienen las restricciones de VaR sobre la selección de la cantidad de contratos forward en un mercado eléctrico y el momento en el que se debe realizar la operación de cobertura, cuando un agente busca maximizar el valor esperado de su beneficio, ajustado por riesgo, y a la vez enfrenta incertidumbre por volumen. Se asume un mercado eléctrico cuyo precio spot presenta características de estacionalidad y reversión a la media y que el precio de los contratos forward exhibe una prima de riesgo (Forward Risk Premium). Como caso de estudio, se presenta el mercado eléctrico colombiano. Los resultados evidencian que las restricciones de VaR logran modificar la razón de cobertura y también el momento en el que se realiza la cobertura.

* Instituto Tecnológico Metropolitano (ITM). Ingeniero Electricista, Facultad de Minas, Universidad Nacional de Colombia; Magíster en Finanzas, Universidad Eafit; Profesor cátedra ITM, Eafit; Especialista en Fundación ECSIM; Consultor independiente en temas de Energía, Modelación Financiera y Riesgos. Correo electrónico: alfredo.trespalacios@gmail. com

† Instituto Tecnológico Metropolitano (ITM). Ingeniero de Producción, Universidad Eafit; Magíster en Finanzas, Universidad Eafit; Profesor tiempo completo, Instituto Tecnológico Metropolitano (ITM); Catedrático, Universidad de Antioquia. Correspondencia: Calle 8 \# 84B-65, apto. 411, Tel.: (+57) 3207253007. Correo electrónico: juanrendon@itm.edu.co.com

$\ddagger$ Universidad Eafit; PhD en Administración; Profesor de la Escuela de Economía y Finanzas, Universidad Eafit. Correo electrónico: jpantoja@eafit.edu.co

Para citar este artículo: Trespalacios, A., Rendón, J. F., \& Pantoja, J. O. (2016). Efecto de Restricciones VaR sobre coberturas en mercados eléctricos. Revista de Economía del Rosario, 19(2), 201-220.

Doi: dx.doi.org/10.12804/revistas.urosario.edu.co/economia/a.5625 
Palabras clave: Mercado eléctrico, cobertura, instrumentos derivados, VaR.

Clasificación JEL: C41, G11, Q40

\title{
Sub-optimal Allocation under VaR Constraints in Electricity Markets
}

\begin{abstract}
We analyze the VaR-constraints effect over decisions about amount and time in the transactions in electricity markets using forward contracts. Taking in account the best hedging time when the markets agents are looking to maximize the expected value of its risk-adjusted utility function and uncertainty faced by volume. We Assume that spot price in electric power market, exhibits especial characteristics such as seasonality and mean reversion. On the other hand electric power market shows evidence of the presence of the risk premium. The results show that VaR restrictions affect the hedging ratio and the time when the hedge is made.
\end{abstract}

Keywords: Electricity Market, Hedging, Derivative Instruments, VaR.

JEL Classification: C41, G11, Q40

\section{Efeito de Restrições de VaR sobre coberturas em mercados elétricos}

Resumo

Analisa-se o efeito que têm as restrições de VaR sobre a seleção da quantidade de contratos forward em um mercado elétrico e o momento no que se deve realizar a operação de cobertura, quando um agente busca maximizar o valor esperado do seu benefício, ajustado pelo risco, e ao mesmo tempo enfrenta incerteza por volume. Assume-se um mercado elétrico cujo preço spot apresenta características de sazonalidade e reversão à media e que o preço dos contratos forward exibe um prêmio de risco (Forward Risk Premium). Como caso de estudo, apresenta-se o mercado elétrico colombiano. Os resultados evidenciam que as restrições de VaR conseguem modificar a razão de cobertura e também o momento no que se realiza a cobertura.

Palavras-chave: Mercado elétrico, cobertura, instrumentos derivados, VaR.

Clasificación JEL: C41, G11, Q40

\section{Introducción}

Las operaciones de coberturas en mercados eléctricos presentan características diferentes a las de otros mercados. Por un lado, la falta de liquidez en las operaciones hacen que las decisiones que toman los agentes sean irreversibles en la práctica; por otro lado, las restricciones de almacenamiento que presenta el activo dificultan la valoración teórica de los productos y hacen que la 
incertidumbre por volumen a transar en spot tenga mayores efectos en el flujo de caja que para otro tipo de activos financieros o bienes primarios.

En la literatura es común encontrar estrategias de coberturas para diferentes productos financieros y commodities en los que el volumen expuesto a riesgo es tratado como una variable determinística. Hull (2009) presenta un esquema de cobertura óptimo por medio de futuros para acciones y divisas donde la fuente de incertidumbre se concentra en los movimientos del precio spot y de los futuros, ignorando la incertidumbre del volumen a transar en spot. Huisman et al. (2007) propone la selección del portafolio de cobertura de agentes de un mercado eléctrico al minimizar el costo de sus compras de energía, pero donde las cantidades de energía a transar son determinísticas.

Näsäkklä y Keppo (2005) consideran la incertidumbre sobre el volumen de energía expuesto a riesgo de spot y proponen un esquema de cobertura utilizando contratos forward, que busca la maximización de la función de utilidad del agente; sin embargo, ignoran la existencia de la prima de riesgo de contratos forward. Esta prima de riesgo se presenta en los mercados eléctricos debido a la incompletitud del mercado y ha sido estudiada por Lucia y Schwartz (2002), Furió y Meneu (2009), Longstaff y Wang (2004) y Pantoja (2009).

La existencia de la prima de riesgo puede generar incentivos para que agentes neutrales al riesgo transen cantidades en contratos forward que pongan en peligro sus pagos ante el mercado, como se explica en Trespalacios et al. (2012), por esto, deben incluirse restricciones adicionales a los esquemas de cobertura al evitar sobreapalancamientos de agentes, en particular, que puedan representar un riesgo sistémico para el mercado. Asimismo, al interior de las compañías, se deberían implementar controles a la gestión de los equipos de negociación, buscando garantizar así que nos e tomen posiciones que puedan poner en riesgo la sostenibilidad de largo plazo de la empresa. El hecho de que las personas encargadas de las transacciones en el mercado tengan el incentivo de maximizar su función de utilidad, y de que sus resultados se evalúen en el corto plazo (digamos un año en el caso del cumplimiento de las metas de la empresa con respecto a presupuestos) puede no ser coherente con las metas de largo plazo de la dirección o de los mismos accionistas.

Para el sector bancario se proponen medidas de control de exposición a riesgo de mercado, basado en el valor en riesgo (VaR por sus siglas en inglés) y exige que este sea calculado diariamente. Mauro (1999) propone implementar el VaR como política de administración de riesgo en el sector energético y explica cómo hacer algunas estimaciones al respecto. En este trabajo se presenta el efecto que tiene la incorporación de una restricción de VaR en la toma de decisiones de cobertura del agente, presentando bajo qué condiciones se encuentran decisiones suboptimas. 
En la sección 1 se describen los modelos matemáticos usados para representar el movimiento del precio del forward. En la sección 2 se analiza la normalidad de la distribución de probabilidad del flujo de caja del agente. En la sección 3 se presenta el VaR del beneficio y su comportamiento ante variaciones en los parámetros; en la sección 4 se presenta la estrategia de cobertura que se recomienda en este trabajo y, finalmente, las conclusiones.

\section{Modelo matemático}

A continuación, se presentan los supuestos acerca de los movimientos del precio de los contratos forward, el volumen que el agente transará en spot (que es el sujeto a la operación de cobertura) en el momento $\mathrm{T}$ y el beneficio obtenido por el agente.

\subsection{Precio forward}

El precio de los contratos forward corresponde al derivado por Lucia y Schwartz (2002) y se presenta en la ecuación 1. Parte del supuesto de que el precio spot Pt es igual a $f(t)+x t$, donde $f(t)$ es una función completamente determinística y $x t$ es un proceso de reversión a la media, con media de largo plazo igual a cero y velocidad de reversión $\mathrm{\kappa}$ como en 2 . El periodo de valoración es $t$, el periodo de madurez $T$ y la prima de riesgo de largo plazo que cobra el mercado es $\alpha$. Como se explica en Lucia y Schwartz (2002), $d w_{x}$ corresponde al diferencial de un proceso de Wiener.

$$
\begin{gathered}
F(t)=f(T)+x_{t} \cdot e^{-\kappa(T-t)}+\alpha\left(1-e^{-\kappa(T-t)}\right) \\
d x=-\kappa \cdot x_{t} \cdot d t+\sigma_{x} \cdot d w_{t}
\end{gathered}
$$

Se aprecia cómo este precio está explicado por tres componentes: una determinística, representada por $f(T)$; la persistencia de las distorsiones de corto plazo, cuyo valor depende de $x_{t} e^{-\kappa(T-t)}$; y la prima de riesgo forward $\alpha\left(1-e^{-\kappa(T-t)}\right)$

A partir de 1, Trespalacios et al. (2012) hallan la ecuación diferencial estocástica 3, que describe el movimiento del precio del contrato forward. Esta ecuación permite encontrar las posibles trayectorias que tendrá el precio forward con vencimiento en $T$ durante el tiempo $t \in\left[t_{t^{\prime}} T\right]$ cuando se cuente con información hasta el momento $t_{0}$. 


$$
\begin{gathered}
d F(t)=\mu_{F}(t) \cdot d t+\sigma \cdot e^{-\kappa(T-t)} \cdot d w_{x} \\
\mu_{F}(t)=-\alpha \cdot \kappa \cdot e^{-\kappa(T-t)}
\end{gathered}
$$

La forma cerrada para el precio forward es:

$$
F(t)=F\left(t_{0}\right)+M_{F}(t)+\int_{t_{0}}^{t} \sigma \cdot e^{-\kappa(T-s)} \cdot d w_{x}(s)
$$

Donde $M_{F}(t)$ es una función determinística que puede ser interpretada como un ajuste a la prima de riesgo forward, que cobra el mercado cuando faltan $T$ - $t$ periodos para el periodo de madurez:

$$
M_{F}(t)=-\alpha\left[e^{-\kappa(T-t)}-e^{-\kappa(T-t)}\right]
$$

Trespalacios et al. (2012) presenta la derivación del comportamiento del precio del contrato forward. La varianza y el valor esperado condicionados en función del tiempo que se presentan en las ecuaciones 7 y 6 . En la figura 1 se presenta una simulación con 100000 trazas del comportamiento del precio para 12 meses adelante, el valor promedio de las trazas y el intervalo de confianza de más y menos una desviación estándar. Para esta simulación, se asumió una prima de largo plazo igual a 10, velocidad de reversión a la media igual a 0,57 y $\sigma=5,19$. Los valores de $\sigma$ у $\kappa$ con los que se hizo dicha simulación fueron calibrados con el precio spot en Colombia. Para el caso de la prima de riesgo de largo plazo, esta se selecciona de forma arbitraria pero coherente con los valores de rangos encontrados por Pantoja (2009). Debido a que $\alpha$ es positiva, se presenta que el precio esperado cae conforme transcurre el tiempo y de acuerdo con la velocidad de reversión esta caída, que es exponencial, será más o menos rápida.

$$
\begin{gathered}
E_{t_{0}}[F(t)]=F\left(t_{0}\right)+M_{F}(t) \\
\text { variance }_{t_{0}}[F(t)]=\frac{\sigma^{2}}{2 \kappa}\left[e^{-2 \kappa(T-t)}-e^{-2 \kappa\left(T-t_{0}\right)}\right]
\end{gathered}
$$




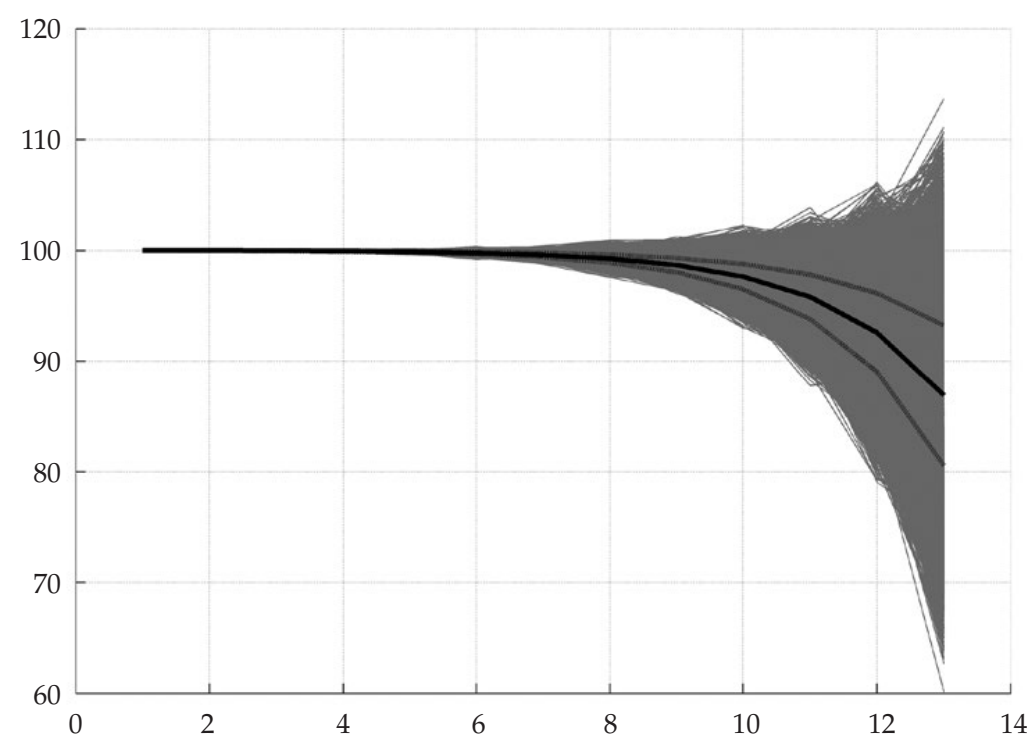

Figura 1. Simulación movimiento del contrato forward con promedio y rango de más y menos una desviación estándar

Eje $x$ : meses, eje $y$ : precio.

Fuente: Elaboración propia.

Así mismo, en la figura 1 se puede ver cómo, a medida que aumenta el tiempo, así lo hará la varianza del precio del contrato forward; en este punto debe hacerse énfasis en que el comportamiento de esta varianza no será igual al comportamiento asumido en la mayoría de los activos financieros, que es una función lineal del tiempo. Por otro lado, el movimiento del precio forward conserva el criterio de convergencia con el spot al momento del vencimiento - este criterio es ampliamente expuesto por Hull (2009) - y, por tanto, la varianza del precio del forward en ese instante de tiempo coincidirá con la varianza del precio spot; de hecho, a nivel general, las distribuciones del precio spot y forward serán idénticas en $\mathrm{T}$. Una vista en tres dimensiones, como la que aparece en 2, permite apreciar cómo el histograma del precio forward se modifica con el transcurrir el tiempo.

\subsection{Volumen transado en spot}

Para cada momento $t$, se asume que la expectativa de volumen a transar (transacción que puede ser asociada con consumo o producción de la energía) por el agente $i$ en el periodo $T$ es $q^{i}(t)$. Näsäkkälä y Keppo (2005) proponen el modelo estocástico que aparece en la ecuación 8. 


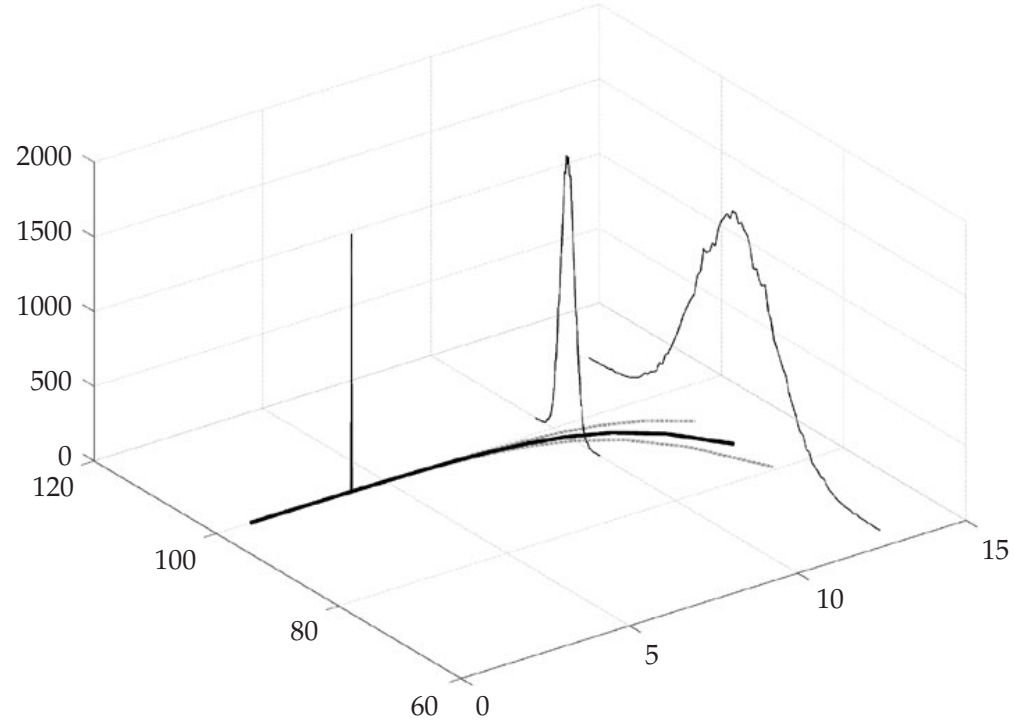

Figura 2. Simulación movimiento del contrato forward con histogramas

Eje $x$ : meses, eje $y$ : precio y eje $z$ : frecuencia absoluta.

Fuente: Elaboración propia.

$$
d q^{i}(t)=q^{i}(t) \cdot\left[\sigma_{q x}^{i} \cdot d w_{x}+\sigma_{q}^{i} \cdot d w_{q}^{i}\right]
$$

Para el instante $t$, la varianza de la estimación de volumen a transar para el agente $i$ es igual a la suma de los cuadrados de los parámetros $\sigma_{q x}^{i} \mathrm{y} \sigma_{q^{\prime}}^{i}$ los cuales son determinísticos y acotados. Para el momento $t_{0^{\prime}}$ la expectativa de volumen inicial es $q^{i}\left(t_{0}\right)=q_{0}^{i}$. La correlación instantánea entre el precio forward y el volumen se presenta en la ecuación 9:

$$
\rho_{q x}^{i}=\sigma_{q x}^{i} \cdot\left[\left(\sigma_{q x}^{i}\right)^{2}+\left(\sigma_{q}^{i}\right)^{2}\right]^{-1}
$$

Así como se hizo para el precio forward, en las figuras 3 y 4 se presentan 1000000 trazas del movimiento de las expectativas de cantidades a transar en spot. Los parámetros del volumen transado dependen de características específicas del agente $i$. 


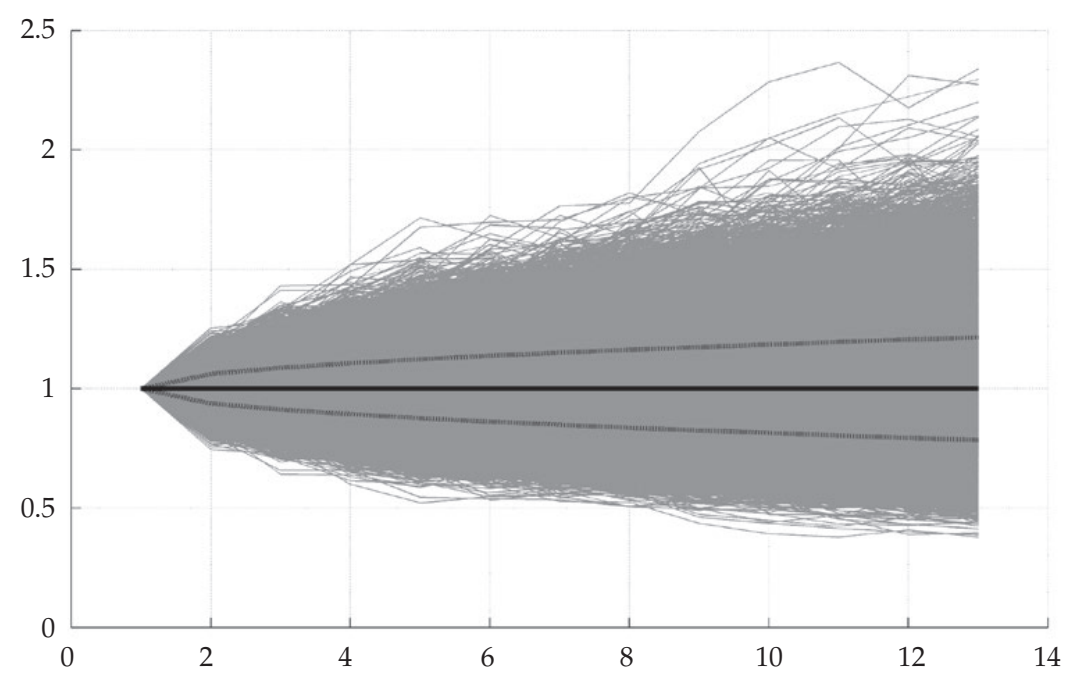

Figura 3. Simulación movimiento de la expectativa de cantidad de energía a transar en spot con rango de más y menos una desviación estándar

Eje $x$ : meses, eje $y$ : cantidad.

Fuente: Elaboración propia.

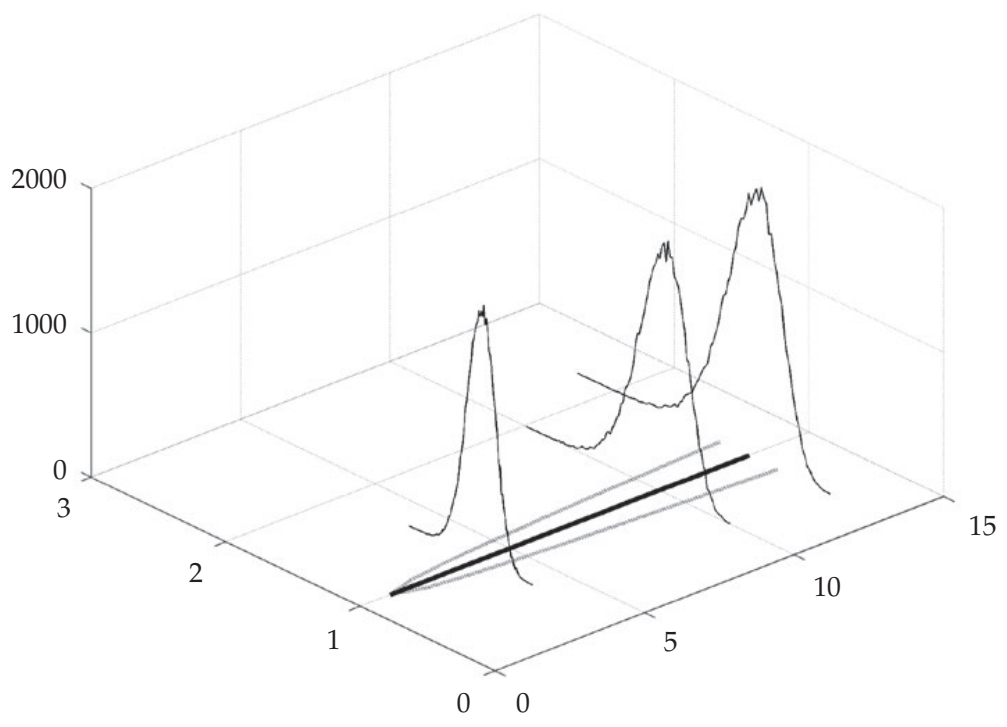

Figura 4. Simulación movimiento de la expectativa de cantidad de energía a transar en spot con histogramas

Eje $x$ : meses, eje $y$ : cantidad y eje $z$ : frecuencia absoluta.

Fuente: Elaboración propia. 


\subsection{Beneficio}

Se asume que los agentes tienen acceso al mercado spot y la cobertura de riesgo se realiza por medio del mercado de contratos forward en el cual solo es aplicable una estrategia del tipo buy and hold. Así, el flujo de caja del agente $i$ para el periodo T está dado por la ecuación 10, en esta se considera el flujo de caja del agente por sus transacciones de energía, tanto en el mercado spot como en contratos forward, que corresponden al primer y segundo, sumando respectivamente. Si un agente no realizara ninguna operación de cobertura por medio del mercado de derivados, su beneficio estaría dado únicamente por $P_{T}$ veces $q^{i}(T)$.

La cantidad de contratos forward que el agente transe en el mercado corresponderá a una proporción $\eta$ de la energía que en el momento que hace la transacción en forward $\tau$ espera transar en spot para el momento $T$, es $\eta \cdot q^{i}(\tau)$.

La función de utilidad S(.) del agente corresponde a la de media-varianza que se explica en Bodie et al. (2009) y aparece en la ecuación 11. La aversión a riesgo del agente está representada en $i$, y para efectos de este trabajo solo se considerarán valores positivos (agentes aversos al riesgo) e iguales a cero (agentes neutrales al riesgo).

$$
\begin{gathered}
\pi^{i}=P_{T} \cdot q^{i}(T)+\eta \cdot q^{i}(\tau) \cdot\left[F(\tau)-P_{T}\right] \\
S(\eta, \tau)=E_{t_{o}}\left[\pi^{i}(\eta, \tau)\right]-\lambda^{i} \cdot \text { variance }_{t_{0}}\left[\pi^{i}(\eta, \tau)\right]
\end{gathered}
$$

\section{La normalidad del beneficio}

Para incorporar las restricciones de $\mathrm{VaR}$ al sistema, se analiza la normalidad del beneficio del agente, para esto se realizó prueba de $\chi^{2}$ a una serie de simulaciones con parámetros de diferentes agentes del mercado colombiano. En ninguna de las pruebas realizadas se logró rechazar la hipótesis nula de normalidad. En la tabla 1 se presentan los resultados a una de las sensibilidades realizadas. Este caso se construyó con $T=12 ; \kappa=0,57 ; \sigma=5,19 ; \rightarrow \rho_{q x}=70 \%$

Debido a los resultados encontrados en esta sección, se considera apropiado el uso de la relación de la ecuación 12, para la estimación del VaR. 
Tabla 1. Prueba de normalidad al beneficio del agente

\begin{tabular}{l|ll|rr|rr|rr|rr|rr|rr}
$\tau$ & \multicolumn{2}{|c|}{$\eta=0$} & \multicolumn{2}{|c|}{$\eta=0,5$} & \multicolumn{2}{|c|}{$\eta=0,8$} & \multicolumn{2}{|c|}{$\eta=0,9$} & \multicolumn{2}{|c|}{$\eta=1$} & \multicolumn{2}{|c}{$\eta=1,1$} & \multicolumn{2}{|c}{$\eta=1,2$} \\
& $\chi^{2}$ & $\mathrm{p}$ & $\chi^{2}$ & $\mathrm{p}$ & $\chi^{2}$ & $\mathrm{p}$ & $\chi^{2}$ & $\mathrm{p}$ & $\chi^{2}$ & $\mathrm{p}$ & $\chi^{2}$ & $\mathrm{p}$ & $\chi^{2}$ & $\mathrm{p}$ \\
\hline 1 & $7 \mathrm{E}-4$ & 1 & 0 & 1 & 0.01 & 1 & 0.01 & 1 & 0.01 & 1 & 0 & 1 & $8 \mathrm{E}-5$ & 1 \\
\hline 6 & $7 \mathrm{E}-4$ & 1 & 0 & 1 & 0 & 1 & 0 & 1 & 0 & 1 & $7 \mathrm{E}-4$ & 1 & $1 \mathrm{E}-4$ & 1 \\
\hline 8 & $7 \mathrm{E}-4$ & 1 & 0 & 1 & 0 & 1 & 0 & 1 & 0 & 1 & $6 \mathrm{E}-4$ & 1 & $4 \mathrm{E}-4$ & 1 \\
\hline 10 & $7 \mathrm{E}-4$ & 1 & 0 & 1 & 0 & 1 & 0 & 1 & $9 \mathrm{E}-4$ & 1 & $8 \mathrm{E}-4$ & 1 & $7 \mathrm{E}-4$ & 1 \\
\hline 12 & $7 \mathrm{E}-7$ & 1 & $7 \mathrm{E}-4$ & 1 & $7 \mathrm{E}-04$ & 1 & $7 \mathrm{E}-4$ & 1 & $7 \mathrm{E}-4$ & 1 & $7 \mathrm{E}-4$ & 1 & $7 \mathrm{E}-4$ & 1
\end{tabular}

\section{VaR del beneficio}

Mauro (1999) describe el VaR (Value at Risk) como la máxima perdida esperada en una actividad, debido a un movimiento adverso en el precio. Si bien, se tomará gran parte de esta definición, se pondrá atención no en los movimientos adversos en el precio sino en los movimientos adversos en el flujo de caja del agente que está interesado en realizar la cobertura. Así, si en épocas de precios bajos se está transando un alto volumen en spot, no será tan perjudicial para el beneficio del agente como aquellos momentos donde se presenten precios bajos y poco volumen de transacciones.

\subsection{Cálculo del VaR}

El Valor en Riesgo puede ser calculado de dos formas: Paramétrica y no paramétrica. Para la primera, Mauro (1999) explica que la metodología más simple corresponde a la del supuesto de normalidad. Para el caso de las no paramétricas, la obtención del percentil correspondiente al VaR puede hacerse mediante simulación de montecarlo y para esto es necesario utilizar la descripción de VaR que propone Meucci (2007).

Para la realización de esta investigación se han utilizado ambas metodologías, pero solo se enuncia en el documento cuándo se realizaron aproximaciones paramétricas bajo el supuesto de normalidad, en caso de que no se especifique la metodología utilizada, el lector debe asumir que fue mediante simulación de montecarlo.

Asumiendo normalidad, con $\sigma_{\pi}$ correspondiente a la raíz cuadrada de la varianza del portafolio al momento de vencimiento, el VaR correspondiente al percentil p \% estará dado por 12 .

$$
\operatorname{VaR}_{p \%}=E[\pi]-z_{p \%} \cdot \sigma_{\pi}
$$


Para estimar el VaR por simulación de montecarlo, se considera que el VaR está dado por el cuantil a cierto nivel, para el caso específico de esta investigación $5 \%$.

\subsection{Sensibilidades al VaR}

En esta sección se presenta el comportamiento del VaR del beneficio del agente ante modificaciones en las variables de gestión de este; de esta forma, el lector podrá generar intuición de cómo se puede mejorar una decisión en términos del VaR asumido. En la figura 5 se muestran varias curvas VaR frente a $\eta$ cuando varía la correlación entre el precio spot y las cantidades; esta simulación se construyó con $\mathrm{F}_{0}=100 \kappa=0,57, \sigma=5,19, \alpha=0, \tau=1, q_{0}^{i}=1$ y desviación estándar de las expectativas de las cantidades al final del periodo $15 \%$.

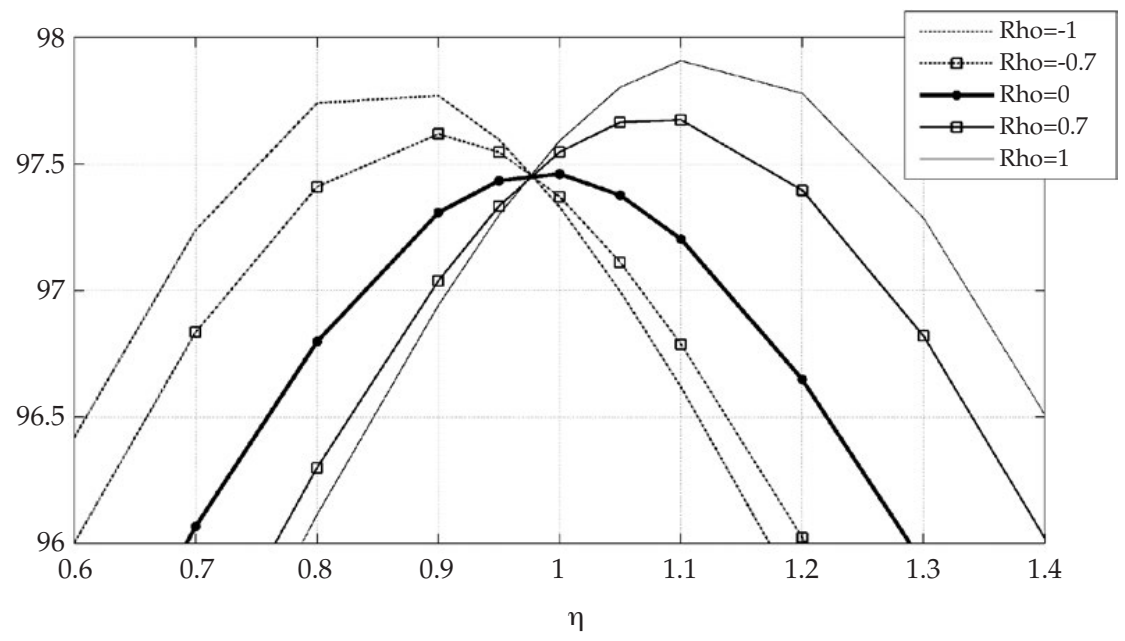

Figura 5. VaR frente a $\eta$ para diferentes correlaciones

Fuente: Elaboración propia.

Cuando la correlación es positiva, se sugiere la necesidad de transar contratos forward por encima de las expectativas de volumen del momento inicial y, cuando la correlación es negativa, se deberán transar contratos forward por debajo de las exceptivas iniciales de cantidades.

En la figura 6 se muestran varias curvas VaR frente a $\eta$, cuando varía a la prima de riesgo cuando la correlación es cero y demás parámetros no especificados corresponden a los de la figura 5. Una prima de riesgo positiva sugiere una transacción en contratos forward, por encima del volumen esperado $(\eta>1)$ y una prima de riesgo negativa sugiere una transacción en contratos 
forward por debajo del volumen esperado $(\eta<1)$ cuando la correlación considerada es cero.

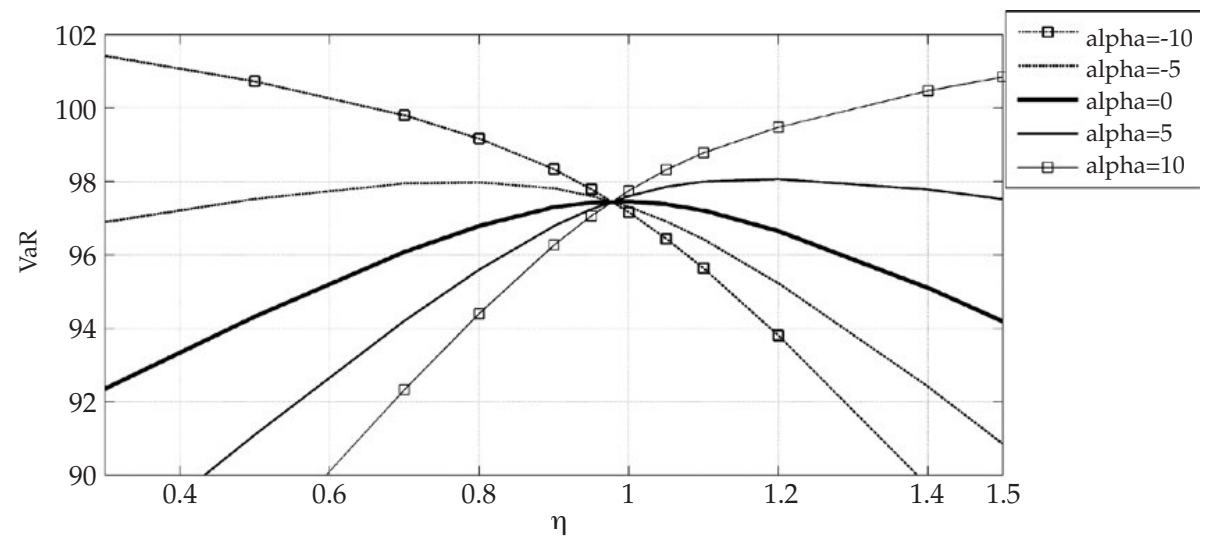

Figura 6. VaR frente a $\eta$ para diferentes primas de riesgo $\alpha$

Fuente: Elaboración propia.

En la figura 7 se presenta la sensibilidad del VaR en el momento en que se hace la transacción $\tau$, la correlación y la prima de riesgo. Si bien, todas estas variables mueven el $\mathrm{VaR}$ del portafolio, se aprecia una alta dependencia de este con la prima de riesgo de largo plazo. Una prima de riesgo positiva sugiere que a medida de que se postergue la operación de cobertura, el VaR caerá, caso contrario ocurre con una prima de riesgo negativa. Si bien, el nivel de correlación afecta el valor del VaR, este no logra modificar la tendencia alcista o bajista de la gráfica VaR frente a $\tau$.

\section{Estrategia de cobertura}

Las variables de gestión, aquellas sobre las que se asume que el agente seleccionará para definir su adecuado nivel de cobertura $\eta$, son la razón de cobertura y el momento en el futuro en el cual se debe hacer la operación de cobertura $\tau$ (timming). Se asume que para el periodo $T$, el agente $i$ busca maximizar el valor esperado $E_{t 0}\left[\pi^{i}\right]$ ajustado por riesgo $-\lambda^{i} \operatorname{Var}_{t 0}\left[\pi^{i}\right]$ de su beneficio, que corresponde a la función de utilidad $S(\cdot)$. De esta forma. $\eta$ y $\tau$ deben ser la solución al problema de optimización planteado en 13. La solución a este problema permite explicar cómo los agentes que presentan una mayor volatilidad en el volumen esperado tienden a postergar la operación de cobertura para 


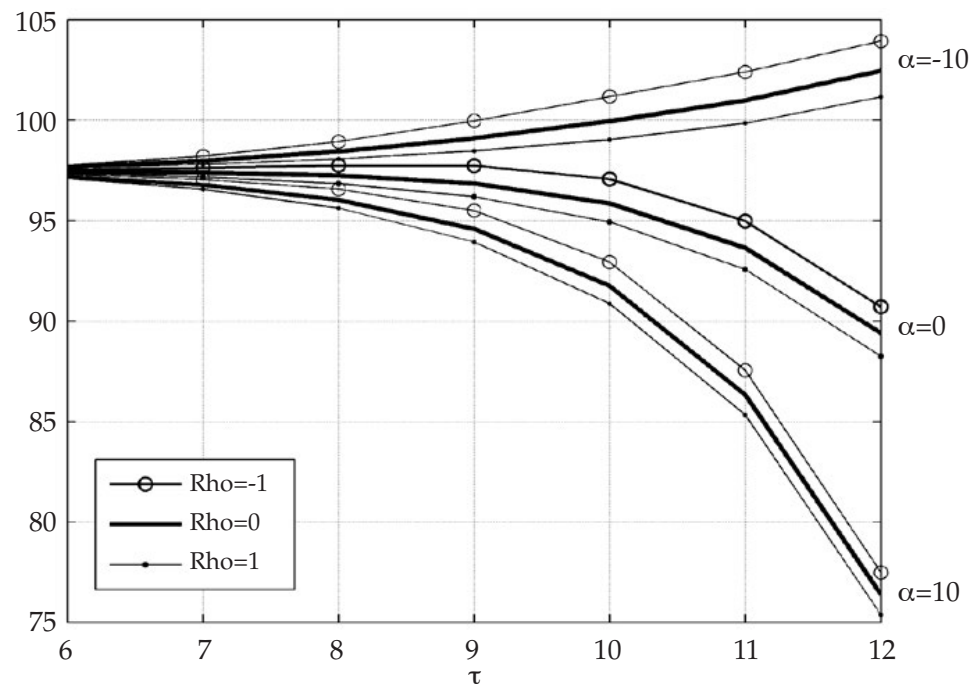

Figura 7. VaR frente a $\tau$ para diferentes correlaciones y prima de riesgo Fuente: Elaboración propia.

lograr mejores estimaciones de su consumo o generación futura y que a medida que aumenta la correlación se incrementa la cantidad necesaria de contratos forward para lograr una adecuada compensación del riesgo asumido. Por otro lado, una correlación negativa es una cobertura natural del flujo de caja del portafolio. Además, cuando la correlación es positiva, la estrategia de cobertura sugiere la anticipación de la transacción. En las simulaciones realizadas para las gráficas que se presentan en esta sección, a menos que se especifique lo contrario, se consideraron los siguientes parámetros que permiten la visualización de algunos comportamientos: $T=12, \rho=70 \% \alpha=5$ y volatilidad total del volumen igual a $5 \%$.

\subsection{Cobertura sin restricciones}

El esquema de cobertura sin restricciones corresponde a la solución del problema de optimización 13.

$$
\max _{\eta, \tau} S=E_{t 0}\left[\pi^{i}\right]-\lambda^{i} \cdot \text { variance }_{t 0}\left[\pi^{i}\right]
$$

Otro resultado importante que subyace al planteamiento del esquema de cobertura sin restricciones es que, dependiendo del signo de la prima de riesgo de largo plazo, un agente estará interesado en anticipar o postergar 
la cobertura; por ejemplo, si la prima de riesgo es positiva, será conveniente vender en contratos forward para el periodo de vencimiento más lejano posible.

En la figura 8 se muestra el conjunto de posibles combinaciones de riesgo $\mathrm{y}$ beneficio para portafolios, dependiendo del valor de $\tau$. El eje vertical corresponde al valor esperado del flujo de caja y el eje horizontal a su varianza. Para cada $\tau$ es eficiente la selección de portafolios que logren un mayor valor esperado que el portafolio obtenido para mínima varianza. Además, no es posible definir un nivel óptimo de cobertura para todos los agentes, ya que el nivel de aversión al riesgo del tomador de decisiones es importante al momento de planear la estrategia; sin embargo, es posible generar un rango de razón de cobertura que cubra un rango de valores de $\lambda^{\mathrm{i}}$. En el caso particular de la figura presentada, se observa que con el hecho de adelantar la cobertura, se pueden obtener portafolios que dominan a los que están más cercanos al momento de maduración. Un agente que posea una posición larga en el subyacente deberá realizar la operación de cobertura en $\tau=1$ y la cantidad de contratos forward dependerá de su nivel de aversión al riesgo.

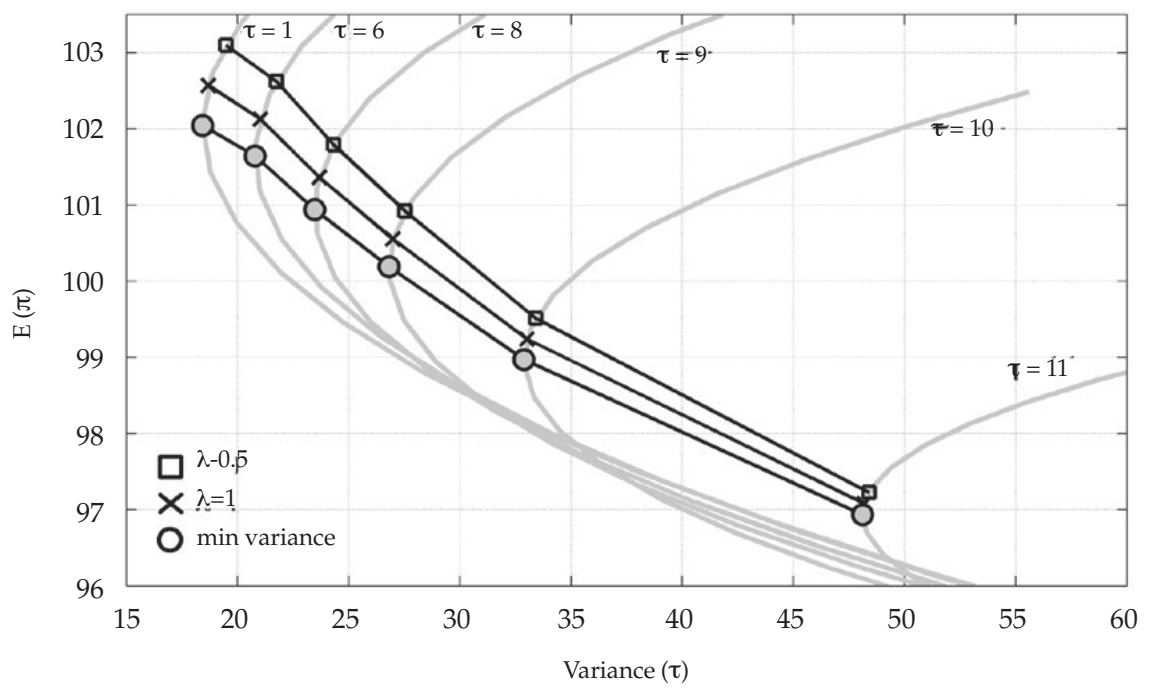

Figura 8. Conjunto de posibles portafolios

Fuente: Elaboración propia.

\subsection{Cobertura con restricción de VaR}

En Trespalacios et al. (2012), se presentó cómo un agente neutral al riesgo —esto es con $\lambda^{\mathrm{i}}=0$ - estaría dispuesto a transar tantos contratos forward 
como le sea posible y el valor esperado de su flujo de caja coincidiría con la prima de riesgo del mercado al momento de realizar la compra o venta de los contratos forward. Esta situación puede convertirse en un incentivo para que algunos agentes no controlen sus posiciones en riesgo y, por tanto, podrían lograr apalancamientos lo suficientemente altos como para exponer el propio mercado a un riesgo sistémico por el no cumplimiento de obligaciones en casos donde lo esperado no sea lo ocurrido, como ha sido el caso en Colombia de algunos comercializadores puros que, al no tener activos de generación de energía, pueden incurrir en pérdidas tan elevadas que lleven a quiebra de la empresa con la posterior irrecuperabilidad de la cartera por los demás agentes del mercado.

Si a la decisión de cobertura se le incorpora una restricción tipo VaR como la que propone Basilea para las corporaciones bancarias, estas no corresponderán necesariamente a la maximización de la función de utilidad del equipo encargado de las transacciones en el mercado eléctrico de cada compañía. En ese caso, la decisión en el esquema de cobertura serán los argumentos que resuelvan el problema 14.

$$
\max _{\eta, \tau} E_{t 0}\left[\pi^{i}\right]-\lambda^{i} \cdot \text { variance }_{t 0}\left[\pi^{i}\right]
$$

s.a.

$$
\operatorname{VaR}_{5 \%} \leq \operatorname{VaR} *
$$

Donde $\mathrm{VaR}^{*}$ es el nivel mínimo de VaR permitido en el portafolio; dicho de otra manera, correspondería con la máxima pérdida esperada permitida para un nivel de confianza.

En la figura 9, la zona sombreada corresponde a la región infactible cuando se selecciona un nivel de $\mathrm{VaR}^{*}=90$; esto es la región en donde posiblemente un agente desee estar ubicado pero por la restricción impuesta en el VaR no le será posible. La curva isoVaR se construye con la ecuación 12 para un percentil del $5 \%$.

A continuación, en la figura 10 se presenta la intersección de las diferentes zonas analizadas. La función de utilidad del agente llevará a que este tome decisiones en la zona a cuadros, que corresponde a aquellos puntos que contienen un valor esperado por encima de la línea de mínima varianza; sin embargo, por la restricción de VaR, solo será adecuado ubicarse en lo que se presenta en la gráfica como la zona de decisión adecuada. 


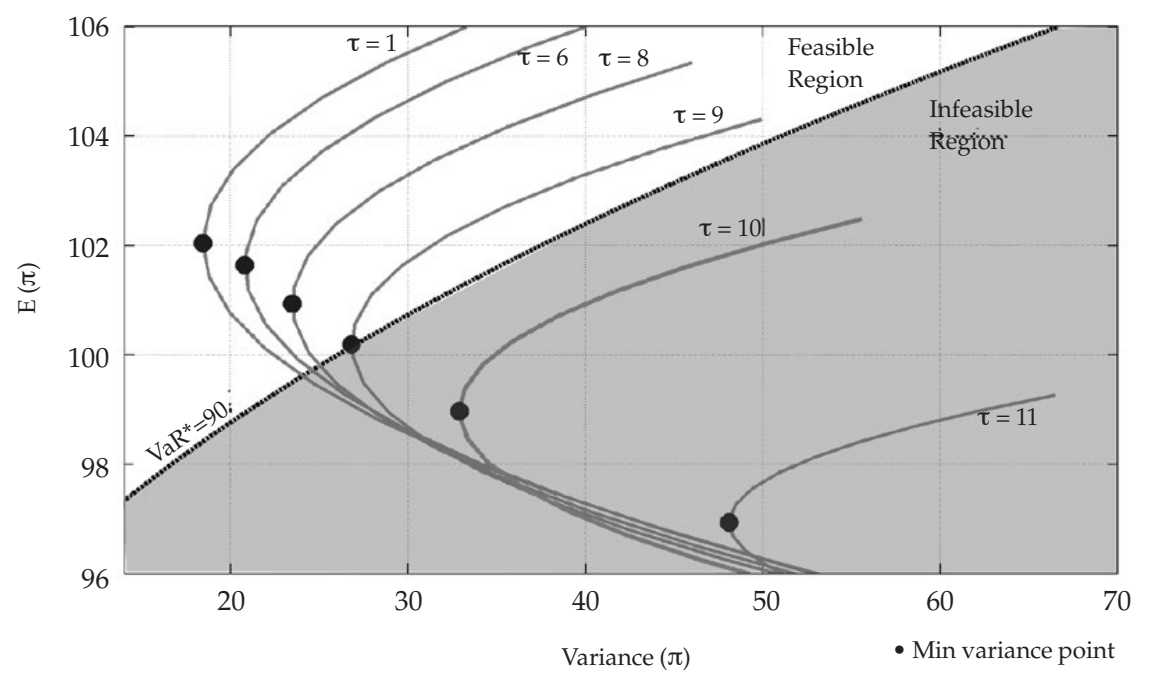

Figura 9. Conjunto de posibles portafolios con regiones factible e infactible Fuente: Elaboración propia.

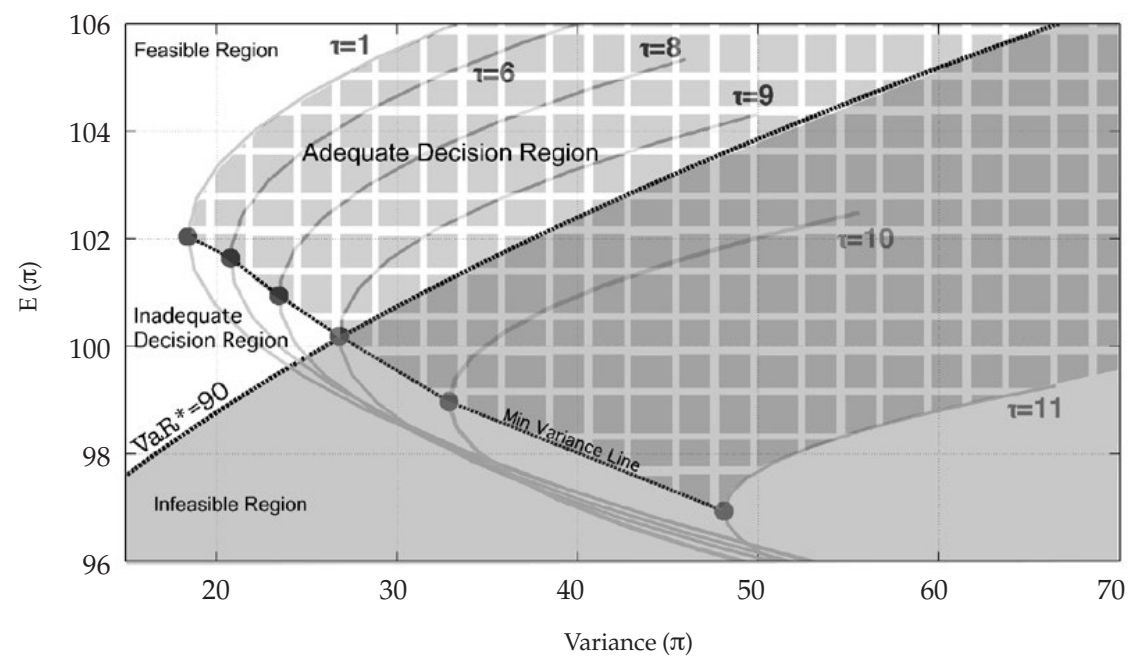

Figura 10. Intersección de región infactible con toma de decisiones sin restricción del portafolio y movimientos en la dirección opuesta a la minimización del riesgo Fuente: Elaboración propia.

En la figura 11 se presentan las posibles combinaciones de riesgo-recompensa que se pueden lograr en el portafolio y diferentes líneas de restricción de VaR. Supongamos un agente que sea averso al riesgo, este tendrá el claro incentivo de seleccionar el portafolio que corresponde a $\tau=1$ y ubicarse en 
el punto de mínima varianza; para este caso, solo el VaR* de 94 se convertiría en una restricción efectiva y forzaría al agente a asumir un poco más de riesgo que el que inicialmente deseaba, claro está que, a cambio, le permite una posición con mayor beneficio. La restricción de VaR puede llevar a los agentes a asumir más riesgo del que desearían, este resultado que puede parecer contraintuitivo se observa principalmente en aquellos casos donde la prima de riesgo es positiva, los equipos encargados de definir políticas de riesgo deberán considerar esta posibilidad al momento de definir restricciones en esquemas de coberturas y definir un nivel de $V_{a} R^{*}$ que mantenga una adecuada flexibilidad para el equipo de gestión de coberturas, ampliando los posibles rangos de $\eta$ y $\tau$ donde poder actuar, pero debe evitar aumentos excesivos en volatilidad y pérdidas importantes en la esperanza.

La forma que logra la figura 11 depende, en principio, de todas las variables involucradas en este documento y, por tanto, lograr una generalización en el esquema de cobertura está por fuera del alcance de esta metodología.

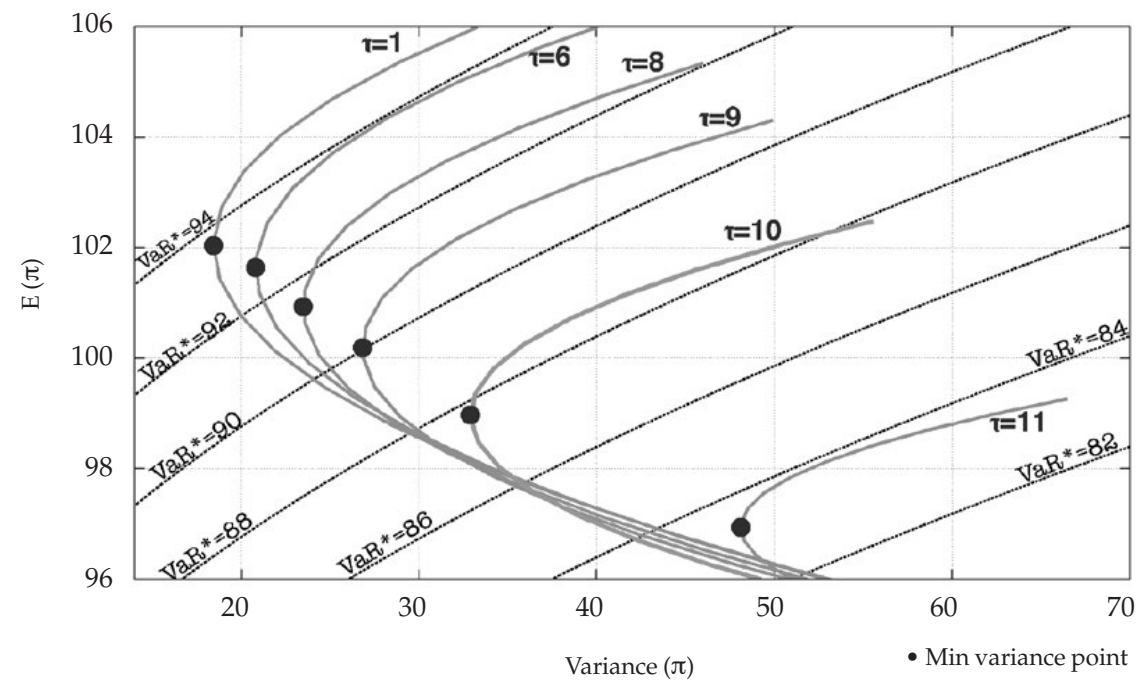

Figura 11. Conjunto de posibles portafolios y líneas de restricción de VaR Fuente: Elaboración propia.

\subsection{Costo de oportunidad}

Cuando el portafolio seleccionado no corresponde a aquel que maximiza la función de utilidad del agente, se dirá, como lo discute ampliamente Meucci (2007), que se está ante una decisión subóptima. Considérese que el vector $d_{\text {opt }}=\left(\eta_{\text {opt }} \mathrm{y} \tau_{\text {opt }}\right)$ corresponde a la solución al problema de cobertura sin 
restricciones, ver ecuación 13 y que el vector $d_{d e c}=\left(\eta_{d e c} \mathrm{y} \tau_{\text {dec }}\right)$ corresponde a la decisión finalmente tomada, que para efectos de este trabajo es la solución del problema de optimización con restricciones, ver ecuación 14. Cuando los vectores $d_{o p t}$ y $d_{d e c}$ no sean iguales, se está presentando que la decisión tomada no corresponde al interés del agente, bien sea porque la decisión final lo está obligando a ubicarse en un punto con mayor varianza o menor utilidad de la inicialmente deseada. A esta pérdida de satisfacción Meucci (2007) la define como el costo de oportunidad (CO), correspondiendo a la resta entre la función de utilidad evaluada en el punto óptimo (deseado) y la función de utilidad evaluada en el punto decidido, como se muestra en la ecuación 15, que para este caso obedece únicamente al efecto de la restricción de VaR.

$$
C O\left(d_{d e c}\right)=S\left(d_{o p t}\right)-S\left(d_{e c}\right)
$$

El costo de oportunidad que se presenta en la ecuación 15 no toma valores negativos, toda vez que $\mathrm{d}_{\text {opt }}$ maximiza el valor de la utilidad $\mathrm{S}(\cdot)$ del agente.

En la tabla 2 se presenta el costo de oportunidad al que se enfrentaría un agente ante diferentes restricciones de VaR; los parámetros para la simulación

Tabla 2. Costo de oportunidad para varios casos $\rho=70 \%, \kappa=0,57, \sigma=5,19, \lambda=1$, $\mathrm{T}=12, \mathrm{~F} 0=100$

\begin{tabular}{|c|c|c|c|c|c|}
\hline VaR $^{*}$ & $\tau$ & $\alpha$ & $S\left(d_{\text {opt }}\right)$ & $S\left(d_{\text {VaRref }}\right)$ & $\mathrm{CO}$ \\
\hline 88 & 1 & -5 & 75.14 & 70.91 & 4.23 \\
\hline 90 & 1 & -5 & 75.14 & 74.74 & 0.4 \\
\hline 92 & 1 & -5 & 75.14 & 41.66 & 33.48 \\
\hline 94 & 1 & -5 & 75.14 & 60.74 & 14.4 \\
\hline 88 & 1 & 5 & 83.94 & 59.05 & 24.89 \\
\hline 90 & 1 & 5 & 83.94 & 67.73 & 16.21 \\
\hline 92 & 1 & 5 & 83.94 & 75.64 & 8.3 \\
\hline 94 & 1 & 5 & 83.94 & 81.52 & 2.42 \\
\hline 88 & 10 & 5 & 66.23 & 60.94 & 5.28 \\
\hline 90 & 10 & 5 & 66.2 & 66.2 & 0 \\
\hline 92 & 10 & 5 & 66.23 & 60.98 & 5.25 \\
\hline 94 & 10 & 5 & 66.23 & 60.98 & 5.25 \\
\hline 94 & 10 & 5 & 66.23 & 33.01 & 33.22 \\
\hline 88 & 10 & -5 & 66.66 & 34.5 & 32.16 \\
\hline 90 & 10 & -5 & 66.66 & 34.5 & 32.16 \\
\hline 92 & 10 & -5 & 66.66 & 34.5 & 32.16 \\
\hline 94 & 10 & -5 & 66.66 & 34.5 & 32.16 \\
\hline 94 & 10 & -5 & 66.66 & 58.02 & 8.64 \\
\hline
\end{tabular}

Fuente: Elaboración propia. 
del precio forward, así como del modelo de volúmenes, son acordes con calibraciones realizadas en el mercado colombiano. $\mathrm{S}\left(\mathrm{d}_{\mathrm{V}}\right.$ aRref $)$ corresponde a la función de utilidad del agente, en caso de tomar una decisión que iguale el VaR de referencia VaR*. Todos estos casos fueron seleccionados de tal forma que la restricción de VaR fuera operativa.

\section{Conclusiones}

En este trabajo se analiza el efecto que tienen las restricciones de VaR sobre el esquema de cobertura que debe plantear un agente que desee maximizar la función de utilidad de media varianza.

En caso de que el esquema de cobertura busque la maximización del VaR, tanto la razón de cobertura como el momento en que se debe hacer la transacción son afectados por la prima de riesgo. Una prima de riesgo positiva sugiere un nivel de transacciones de contratos forward por encima del volumen esperado y tiende a adelantar la cobertura. Una prima de riesgo negativa sugiere retrasar la operación de cobertura y una menor transacción en contratos forward que el volumen esperado. Si bien, el nivel de correlación afecta el valor del VaR, este no logra modificar la tendencia alcista o bajista de la gráfica VaR frente $\tau$.

Cuando la correlación es positiva, se sugiere la necesidad de transar contratos forward por encima de las expectativas de volumen del momento inicial y, cuando la correlación es negativa, se deberán transar contratos forward por debajo de las exceptivas iniciales de cantidades.

La restricción de VaR logra modificar no solo la razón de cobertura sino también el momento cuando se realiza la cobertura, hablar de cuando la prima de riesgo es positiva y de cuando la prima de riesgo es negativa.

Una vez se ha definido el VaR mínimo de referencia, el costo de oportunidad de los agentes dependerá de la situación en la que se encuentra el mercado de contratos forward, afectándose en gran medida de la prima de riesgo.

\section{Referencias}

Bodie, Z., Kane, A., \& Marcus A. J. (2009). Investments. Chicago: McGraw Hill. Furió, D., \& Meneu, V. (2009). Expectations and forward risk premium in the spanish deregulated power market. Working Paper, Instituto Valenciano de Investigaciones Económicas, S.A. (Ivie) 
Huisman, R., Mahieu, R., \& Schlichter, F. (2007). Hedging exposure to electricity price risk in a value at risk framework. Erim Report Series Research in Management.

Hull, J.C. (2009). Options, Futures and other Derivatives. Nueva York: Pearson Prentice Hall.

Longstaff, F. A., \& Wang, A. W. (2004). Electricity Forward Prices: A HighFrequency Empirical Analysis. The Journal of Finance, 59, 1877-1900.

Lucia, J., \& Schwartz, E. (2002). Electricity prices and power derivatives: Evidence from the Nordic Power Exchange. Review of Derivatives Researh 5, 5-50.

Mauro, A. (junio, 1999). Price risk management in the energy industry: The value at risk approach. XXII Annual International Conference of the International Association for Energy Economics, Rome.

Meucci, A. (2007). Risk and Asset Allocation. Nueva York: Springer Finance.

Näsäkkälä, E., \& Keppo, J. (2005). Electricity load pattern hedging with static forward strategies. Managerial Finance vol 31, 116-137.

Pantoja, J. O. (2009). Modelling risk of electric power markets. Working Paper, Universidad EAFIT.

Trespalacios, A., Rendón, J. F., \& Pantoja, J. O. (2012). Forward contracts based on hedging strategy in electric power markets. Academia, Revista Latinoamericana de Administración, 50, 148-157. 